\title{
Aplicação da Metodologia Multicritério de Apoio à Decisão no Relacionamento Interorganizacional na Cadeia da Avicultura de Corte $^{1}$
}

\author{
Ângelo Brambila Reck² e Glauco Schultz ${ }^{3}$
}

Resumo: A avicultura de corte é uma importante fonte de proteína para a alimentação humana. A compreensão do relacionamento interorganizacional neste ambiente é primordial para a redução de custos operacionais e de transação. Este trabalho objetiva construir um modelo multicritério de avaliação do relacionamento entre uma agroindústria e seus produtores integrados, em Seara, Santa Catarina, Brasil, avaliando-se os possíveis reflexos sobre a competitividade da cadeia de suprimentos. Realizou-se um estudo de caso tendo como enfoque teórico a gestão da cadeia de suprimentos, complementada pela abordagem economia dos custos de transação e das estratégias competitivas. A pesquisa foi quanti-qualitativa e utilizou-se da metodologia de análise multicritério de apoio à decisão construtivista (MCDA-C) para elaboração e interpretação do modelo. Realizaram-se entrevistas com produtores rurais, representantes da agroindústria e especialistas da avicultura. Os agentes definiram custo de produção, remuneração, fatores de produção, risco de investimento, fluxo de informação e ambiente institucional como os pontos de vista fundamentais (PVF) do modelo. Com a aplicação do modelo em três classes de produtores rurais, os PVFs remuneração, risco de investimento, fluxo de informação a jusante da cadeia de suprimentos e custo de produção apresentaram maior impacto no relacionamento interorganizacional para se atingir a competitividade na cadeia.

Palavras-chaves: Agronegócios; Avicultura de corte; Cadeia de suprimentos; Relacionamento interorganizacional; Multicritério.

Abstract: The poultry production is an important source of protein for human consumption. Understanding the inter-relationship in this environment is essential to reduce operating and transaction costs. This work aims to build a multi-criteria evaluation model of the relationship between an agribusiness

1. Data de submissão: 23 de agosto de 2015. Data de aceite: 14 de agosto de 2016.

2. Universidade Federal do Rio Grande do Sul. Porto Alegre. Rio Grande do Sul. Brasil. E-mail: angelotche@yahoo.com.br

3. Universidade Federal do Rio Grande do Sul. Porto Alegre. Rio Grande do Sul. Brasil. E-mail: glauco.schultz@ufrgs.br 
and its integrated producers in the city of Seara, Santa Catarina, evaluating the possible effects on the competitiveness of the supply chain. We conducted a case study with theoretical approach to supply chain management, complemented by microanalytical approach of competitive strategies. It conducted quantitative and qualitative research and used the multi-criteria analysis methodology to support constructivist decision (MCDA-C) for the construction and interpretation of the model. We used instrument and carried out interviews with farmers, agribusiness representatives and poultry specialists. The agents defined production cost, remuneration, factors of production, investment risk, information flow and institutional environment as the fundamental points of view (FPV) model. It was found with the application of the model in three classes of farmers that FPV's remuneration, investment risk, downstream flow of information supply chain and production costs, had greater impact on the inter-relationships to achieve competitiveness in chain.

Key-words: Agribusiness; Poultry production; Supply chain; Interorganizational relationship; Multi-criteria.

DOI - http://dx.doi.org/10.1590/1234-56781806-94790540407

Classificação JEL: Q13.

\section{Introdução}

O processo de industrialização da agricultura provocou modificações nas relações interorganizacionais no âmbito das cadeias produtivas agroindustriais, sendo que estas passaram a ser verticalizadas, demandando recursos tecnológicos, infraestrutura e principalmente uma gestão compartilhada dos processos. Dessa forma, passou-se a depender de estratégias articuladas pelo setor, baseadas nas condições internas e externas do mercado e da capacidade em atender as demandas dos stakeholders. Os agentes econômicos que fazem parte de uma cadeia de suprimentos buscam gerar valor para diferenciar seus produtos em um mercado altamente competitivo. Para isso é necessário que as empresas busquem eficiência e eficácia nos seus processos, que pode ser traduzida pela redução nos custos de produção e de transações e por ações inovativas para diferenciação perante os concorrentes. Evidencia-se, a partir dessas afirmações, a necessidade de um planejamento e gerenciamento conjunto na cadeia de suprimentos para o alcance de melhores resultados dos agentes econômicos. O gerenciamento dos arranjos interorganizacionais cria um fortalecimento dos vínculos entre os segmentos e uma reciprocidade nas ações, o que leva a ganhos mútuos.
O presente artigo apresenta os resultados de um estudo de caso realizado na cadeia de suprimentos da avicultura de corte ${ }^{4}$, buscando interpretar as relações interorganizacionais para a construção de indicadores de competitividade, por meio de uma metodologia de análise robusta e consistente que consiga identificar os fatores qualitativos e quantitativos dos relacionamentos, considerando a seguinte questão de pesquisa: como os agentes da cadeia de suprimentos da avicultura de corte se relacionam e que critérios poderiam auxiliar na tomada de decisão dos atores e desta forma estreitar o relacionamento? Para responder a essa questão foi elaborado um modelo multicritério de avaliação do relacionamento interorganizacional entre uma agroindústria e seus produtores integrados, com os possíveis reflexos sobre a competitividade na cadeia da avicultura de corte. Para isso foram definidas variáveis e construídos indicadores que permitiram aplicar o modelo e avaliar a competitividade de um caso de integração na avicultura de corte no Brasil.

A avicultura de corte é considerada uma importante cadeia de suprimentos no setor agroindustrial, com expressivos investimentos tecnoló-

4. Avicultura de corte neste artigo trata exclusivamente da cadeia de suprimentos de frango de corte. 
gicos, de capital e geração de empregos. Possui vantagens competitivas devido ao rápido ciclo produtivo, ter a possibilidade de uma estrutura organizacional verticalizada e ser uma proteína animal de baixo custo, o que atrai consumidores de diferentes classes sociais, quando comparados a outros setores do agronegócio. A análise da competitividade constitui-se como o principal meio em que uma empresa obtém vantagens sustentáveis na sua área de atuação, servindo de parâmetro para a concorrência em determinado mercado. A análise da cadeia de suprimentos é o direcionador para obtenção de valor, uma vez que permite aos agentes atuarem de forma sinérgica, com ações planejadas, devidamente organizadas e coordenadas. Assim, a análise de como a cadeia gerencia seus segmentos permite avaliar o desempenho efetivo dos agentes, monitorar a atuação frente aos concorrentes e preconceber ações corretivas, e assim possibilitar mensurar o efeito competitivo. Para responder a estas questões buscou-se embasamento teórico nas abordagens de Gestão da Cadeia de Suprimentos, Estratégias Competitivas e Economia dos Custos de Transação, para assim compreender os direcionadores que possibilitam a geração de valor e a redução nos custos.

O presente artigo está dividido em quatro seções, além desta introdução. Na próxima seção consta a apresentação da fundamentação teórica utilizada para conduzir a investigação. A seção três contém a metodologia de estudo e na seção quatro encontram-se a descrição do modelo multicritério e os resultados obtidos com a sua aplicação. A última seção é constituída das conclusões do estudo.

\section{Fundamentação teórica}

A presente seção tem por objetivo apresentar as três abordagens teóricas que foram utilizadas para construção do modelo multicritério de avaliação do relacionamento interorganizacional entre uma agroindústria e seus produtores integrados: gestão da cadeia de suprimentos (supply chain management), economia dos custos de transação e estratégias competitivas. Primeiramente discorre-se sobre cada uma das abordagens e posteriormente busca-se realizar a apresentação da metodologia multicritério, que será utilizada para construir o modelo, integrando assim as três abordagens teóricas.

\subsection{Gerenciamento da cadeia de suprimentos}

Um novo contexto global está modificando a percepção das firmas quanto à competitividade, pois estas estão priorizando organizar-se em cadeias de suprimentos. Assim, para as empresas a aproximação com os fornecedores e clientes vem sendo uma estratégia recorrente no sentido de organizar e coordenar a cadeia de suprimentos, integrando os agentes de tal forma que se assemelhe a uma grande firma (FURLANETTO, 2002). O significado de cadeia de suprimentos é retratado por Van der Vorst e Beulens (2002, p. 2) como "o planejamento integrado, coordenado e com o controle de todos os processos e atividades do negócio na cadeia de suprimentos para a maximização de valor, a um custo mínimo ao consumidor final. $\mathrm{O}$ conceito remete à relação de interdependência entre os agentes, permitindo analisar o que a cadeia de suprimentos possibilita realizar para a empresa e quais as implicações das ações conjuntas com fornecedores e clientes para o atendimento das necessidades dos consumidores.

A cadeia de suprimentos tem em seus princípios a agregação de valor com a sucessão de etapas e a organização das firmas de forma verticalizada. Assim são identificadas três fontes principais de valor: a otimização da produção e operação, que consiste em uma ferramenta de planejamento que busca desenvolver o sistema como um todo, em uma visão integrada da empresa, estendendo-se ao conceito de gestão logística; a redução dos custos de transação, via otimização das transações com base na percepção de que existem custos positivos na utilização do sistema no mercado e a apropriação dos direitos de propriedade, ou seja, pela manutenção dos 
712 - Aplicação da Metodologia Multicritério de Apoio à Decisão no Relacionamento Interorganizacional na Cadeia da Avicultura de Corte

benefícios oriundos da inovação (LAZZARINI et al., 2001).

Dessa forma, o que torna a cadeia de suprimentos um mecanismo diferenciado são suas particularidades, como apontam Lambert e Cooper (2000) que destacam: as muitas etapas de crescimento intraorganizacional e interorganizacional, referindo-se à coordenação vertical; interdependência entre as firmas, indicando que os relacionamentos entre os agentes é fundamental; apresentam fluxo bidirecional de produtos, informações, gestão e atividades operacionais e o atendimento dos objetivos coletivos da cadeia propicia a criação de valor para o cliente e a otimização da utilização dos recursos disponíveis. Estes atributos agem sobre a eficiência ao longo do canal de distribuição permitindo o planejamento e a gestão conjunta entre seus diversos agentes, pois tem como foco a coordenação e a integração de atividades relacionadas ao fluxo de produtos, serviços e informações entre os diferentes elos.

Neste contexto, a interdependência de atividades é um processo vital para a entrega de um produto ou serviço ao consumidor final, pois se uma atividade falhar, a cadeia se desconectará, ocasionando um desempenho insatisfatório e este desestabilizará a carga de trabalho em outras áreas, bem como exigirá um novo planejamento (STEVENS, 1989; VAN DER VORST; BEULENS, 2002). As informações incompletas e assimétricas em situações de interesses comuns levam a custos de coordenação. Contudo, este problema pode ser resolvido pela escolha de um mecanismo adequado que alinhe os interesses, tais como preço, acordos contratuais ou autoridade (BIJMAN, 2002, p. 24).

O desafio da coordenação, segundo Guanziroli et al. (2008, p. 17) é "definir e operar mecanismos, seja por incentivos econômicos, regulatórios e contratuais que mitiguem os conflitos, contradições e custos de transação ao longo de toda a cadeia" e, conjuntamente, reiterem o apoio dos diferentes agentes com os objetivos estratégicos dos líderes do setor, reduzindo, dessa forma, os custos de monitoramento.
Como atributos que podem ser sinalizados para a construção de indicadores de competitividade estão a identificação dos agentes, a avaliação de ações conjuntas, a consideração sobre uma visão de longo prazo, a liderança do setor, o fluxo de informações, a padronização de ações, a construção de uma marca para a cadeia, o compartilhamento dos lucros e a velocidade das transações (ARBAGE, 2004).

\subsection{Economia dos custos de transação}

A economia dos custos de transação constitui-se como parte da Nova Economia Institucional, contribuindo para avaliar as ações e interações das firmas influenciadas por instituições. As normas sociais, políticas e legais são representadas pelo ambiente institucional, constituindo-se como a base para a produção e as relações das empresas, bem como a normatização de direitos, como o de propriedade, contratos, entre outros (WILLIAMSON, 1993; ZYLBERSZTAJN, 1995). A teoria dos custos de transação, segundo Coase (1988), propõe que a firma tenha como função economizar os custos de transação, o que se realizaria de duas maneiras: por meio do mecanismo de preços, que possibilitaria à empresa escolher os mais adequados em suas transações com o mercado; e substituindo um contrato incompleto por vários contratos completos, uma vez que seria de se supor que contratos incompletos elevariam custos de negociação.

Farina et al. (1997) apresentam quatro fontes de custos de transação: 1) os custos para construção e negociação dos contratos; 2) os custos de mensuração e monitoramento do direito à propriedade referenciados nos contratos; 3) os custos para manter e executar os contratos, sejam eles internos ou externos à empresa e 4) os custos de adaptação a um novo ambiente. A superação pelas organizações destas barreiras incide em um menor custo de transação, tornando, assim, a empresa mais competitiva nos mercados em que atua.

As estruturas de governança específicas surgem devido ao caráter incompleto dos contratos, 
que deixam os agentes econômicos em situação de insegurança jurídica. O risco do não cumprimento contratual exige a necessidade de garantias das instituições controladoras que avalizam o cumprimento do contrato. O destino é uma estrutura de governança mais adaptada a determinadas circunstâncias ambientais, que implicam em um menor custo de transação em relação a um melhor investimento de recursos (DOROW, 2013). Assim, a governança é considerada como "o esforço para habilitar a ordem, e com isso mitigar conflitos e aumentando o ganho mútuo" (WILLIAMSON, 2000, p. 599 apud BIJMAN, 2002), além de permitir a alocação de direitos de decisão e direito de renda sobre todos os ativos.

A estrutura de governança pode ser formada via mercado (spot), híbridas ou hierárquica, sendo que estas estruturas se diferenciam prioritariamente conforme o nível de controle e incentivos que determinados agentes exercem uns sobre os outros. A presença de mecanismos contratuais de governança distingue-se em formais e informais. Os mecanismos formais incluem a integração vertical e os contratos (formais) e os mecanismos de governança informal incluem acordos, confiança, comprometimento e reputação (TRIENEKENS et al., 2012). Para estes autores, os contratos formais representam um mecanismo comum de governança, em que se destacam elementos como: qualidade do produto, por meio de normas; condições de entrega, em função do tempo; preço; ordem de frequência e de tempo; condições de pagamento; especificidades de armazenamento e transporte; embalagem; origem e rastreabilidade; marketing; sanções no caso de não cumprimento e duração do contrato. Também aspectos como a confiança, satisfação e comprometimento são variáveis utilizadas para avaliar os relacionamentos em uma cadeia produtiva.

\subsection{Estratégias competitivas}

Nos sistemas agroindustriais, diversos autores (VAN DUREN et al., 1991; FARINA et al., 1997; SILVA e BATALHA, 1999; BATALHA e SOUZA FILHO, 2009) discorrem sobre a importância da competitividade para as firmas, sendo que esta pode ser mensurada pela capacidade de ganhar e preservar parcelas de mercado. Para tal, é necessário percorrer três vias principais: a otimização da economia de escala (mínimo do custo), da economia de escopo (em uma mesma planta produtiva ter mais de um produto e/ou serviço) e de eficiência nas transações (redução dos custos de negociação). Assim, a competitividade dos agentes econômicos é condicionada pelas ameaças e oportunidades que afetam as suas atividades e o seu posicionamento no mercado, caracterizando, assim, o conceito de estratégia competitiva (KATZ, 1970; ANSOFF, 1977).

O termo competitividade é amplamente utilizado com o objetivo de representar medidas de desempenho, sendo uma das definições a capacidade da firma em formular e implementar estratégias concorrenciais, que lhe permitam ampliar ou conservar, de forma duradoura, uma posição sustentável no mercado. Decorre dessa definição, por exemplo, que para uma empresa ser competitiva, deverá adequar as suas estratégias individuais ao padrão de concorrência vigente no mercado específico (FERRAZ et al., 1995). Neste contexto, a competitividade é caracterizada como uma medida de desempenho das firmas e que está envolta por relações intra (custo de produção) e entre organizações (custo de transação e arranjos de coordenação). Já a definição de estratégia está relacionada à possibilidade de criar e capturar valor, de acordo com o posicionamento estratégico das empresas condicionado pelas forças setoriais exercidas pelos concorrentes, compradores, fornecedores, novos entrantes e produtos substitutos (PORTER, 1985).

A implementação de estratégias competitivas pelos agentes de uma cadeia de suprimentos está diretamente relacionada às capacidades internas da firma e ao grau de pressões externas. A busca por atributos diferenciados passa a ser o objetivo para galgar vantagens competitivas, sendo os principais modelos: (a) integração vertical (processamento da produção e formação de marca); (b) contratos (relações formais entre o segmento rural com o industrial); (c) ações conjuntas (união 
714 - Aplicação da Metodologia Multicritério de Apoio à Decisão no Relacionamento Interorganizacional na Cadeia da Avicultura de Corte

de produtores em torno de atributos diferenciados da região) (SAES, 2008). Na perspectiva da Visão Baseada em Recursos (VBR), a obtenção de vantagens competitivas sustentáveis é pautada por estratégias internas de criação de valor, que permitem a alocação de recursos de forma distinta dos outros competidores ou que outra empresa não consiga reproduzir os benefícios da estratégia adotada pelo concorrente (BARNEY, 1991). Com relação ao ambiente externo em que as organizações estão inseridas, este apresenta mudanças que trazem pressões competitivas, devido aos novos concorrentes e à transnacionalização das cadeias produtivas, pressões tecnológicas, advindas de inovações, novos métodos de produção, pressões financeiras, com redução permanente de custos, pressões políticas, como a emergência de conflitos, pressões em termos de saúde, com a maior preocupação com obesidade, valor nutricional, facilidade digestiva e finalmente, pressões de valor, com as emergentes questões éticas e de bem-estar (NEVES e NEVES, 2011, p. 46).

\subsection{Metodologia multicritério de apoio à decisão}

Dentre as diversas propostas de avaliação e ferramentas de mensuração, a Pesquisa Operacional (PO) constitui-se como um notável meio de análise para alocação de recursos escassos nas esferas organizacionais e interorganizacionais, além de permitir avaliar os riscos decorrentes da atividade que interferem sobre a competitividade (KALOGERAS et al., 2005). A PO é uma área do conhecimento que se utiliza de meios científicos para a resolução de problemas, partindo do pressuposto da construção de modelos decisionais (ROY, 1993). Esses modelos constituem-se de um retrato particular da realidade, demonstrados através de funções matemáticas, sistemas, árvores de decisão, programação linear ou mapas cognitivos (ENSSLIN et al., 2001; XAVIER et al., 2012). Para Reichert (2012), as metodologias de análise multicritério (MCDA - MultiCriteria Decision-Aid) compõem parte do arcabouço da $\mathrm{PO}$, tanto pelo apoio à decisão proposto pela abordagem multicritério, como pela tomada de decisão, marca característica da PO tradicional. $\mathrm{O}$ que as distingue é o enfoque sobre o problema empregado. Para a MCDA, o relevante é a visão do(s) decisor(es), composta de incertezas e limitações, em que é primordial a consideração dos valores, objetivos, cultura, preconceitos etc. dos agentes envolvidos na decisão (ROY e VENDERPOOTEN, 1996). Esta interação por parte dos atores permite um conhecimento mais aprofundado acerca do problema e gera a construção objetiva e representativa do modelo e dos critérios (ENSSLIN et al., 2001).

No contexto dos sistemas agroindustriais, a utilização de ferramentas de Apoio à Decisão torna-se uma alternativa robusta para harmonizar os interesses dos diferentes agentes e permitir uma avaliação mais segura de desempenho das cadeias. Trabalhos são encontrados referentes a sistemas de produção agropecuários (MAZZETTO e BONERA, 2003; GOMES et al., 2008; REICHERT, 2012); planejamento de sistemas de cultivo (LOYCE et al., 2002; AREND et al., 2011; XAVIER et al., 2012); escolha de cultivares (MASTRANTONIO et al., 2007); aspectos ambientais (ARONDEL e GIRARDIN, 2000; GENERINO, 2006); relações em um sistema de integração de frango de corte (BALVERDE, 2006); performance de agroindústrias (GOMES, 2001); mercado agropecuário (KALOGERAS et al., 2005; ALMEIDA, 2011); avaliação do potencial de negociação das tecnologias geradas com Empresas de Base Tecnológica (MARTINS et al., 2011) e gestão da inovação do conhecimento em arranjos produtivos locais (BELARMINO et al., 2011).

Para Ensslin et al. (2001), esta ferramenta estabelece suporte à tomada de decisão, diante de problemas complexos que buscam soluções satisfatórias para os agentes, em que exige a interlocução de um facilitador e um decisor. A MCDA, em seu sentido construtivista, participa na estruturação de modelos decisionais, a partir da construção do conhecimento entre os agentes, por meio de seus valores e objetivos, tendo como princípio fundamental a busca da solução mais apropriada para os participantes ao invés de uma solução 
matemática ótima (ROY, 1996). Neste contexto, a metodologia pauta-se pelo paradigma construtivista, que indica o constante aprendizado e evolução sobre a prática decisória. Além de alicerçar dois princípios básicos: a possibilidade do decisor de solucionar questões pela comparação de critérios conflitantes e facilitar o processo de escolha, ordenação, classificação ou descrição das alternativas conforme o seu impacto (ROY, 1996). Assim, o escopo de atuação da MCDA-C centra-se em três pontos: a) a participação dos envolvidos no processo decisório (decisores), permitindo a profunda compreensão da evolução do processo e o julgamento conforme seus valores e objetivos; b) a presença de um facilitador para auxiliar no processo de construção do conhecimento do decisor e modelagem do sistema; c) e a busca da solução mais adequada conforme a percepção e valores dos decisores (DUTRA et al., 2008). Para Ensslin et al. (2001), o método destaca-se pelo fato de considerar a subjetividade dos decisores e possibilitar uma abordagem multidisciplinar, assim, exige-se uma mobilização coletiva dos envolvidos na construção de todas as etapas do processo para avaliarem as alternativas, uma vez que consistirá na emergência dos problemas a serem resolvidos e quais parâmetros serão utilizados para avaliação das alternativas.

\section{Metodologia}

Adotou-se o estudo de caso com múltiplas unidades de análise (YIN, 2001) como estratégia de pesquisa nas propriedades rurais e na agroindústria integradora e a realização de entrevistas semiestruturadas e de reuniões como procedimentos de campo para estudo da cadeia de suprimentos da avicultura de corte. A pesquisa foi desenvolvida em três etapas distintas: estruturação, avaliação e recomendação.

A primeira etapa foi a de estruturação, em que ocorreu a definição do problema de pesquisa, foram identificados os agentes envolvidos, bem como foi realizada a operacionalização dos elementos para construção do modelo (REICHERT,
2012). Desta fase foram obtidos os pontos de vista que compõem os objetivos e valores dos agentes, sendo estes os elementos que embasaram o modelo de avaliação multicritério. Esta fase ocorreu entre maio e julho de 2014 e constituiu-se no levantamento e análise das informações obtidas com a aplicação de um instrumento (roteiro de entrevistas) envolvendo a participação de representantes da agroindústria integradora (gerente agropecuário e supervisor de fomento) e nove produtores integrados, distribuídos nos municípios de Seara, Itá, Xavantina, Arvoredo e Paial, na região meio-oeste do estado de Santa Catarina. Foram levantadas informações sobre: os riscos e estratégias para atingir a competitividade na propriedade, a existência de informações incompletas nas transações e as possíveis formas para a resolução, a visão de longo prazo do relacionamento, a relação com as especificidades de ativos, as incertezas e a frequência com que se relacionavam, a existência de racionalidade limitada e de ações oportunistas decorrentes da incompletude de informações. O grupo de produtores rurais selecionados para participar da formulação do modelo multicritério tinha como características: a) perfil empreendedor, com baixo custo de produção, ou seja, resultados próximos às metas estabelecidas pela empresa, especialmente em conversão alimentar; b) atividade constituía-se como uma das principais fontes de renda para a propriedade e c) possuía escala de produção ${ }^{5}$ ou área para expansão. Esse diagnóstico inicial teve como finalidade a busca de informações que apoiassem a construção do modelo multicritério para avaliação do relacionamento. Para isso, as entrevistas foram transcritas e identificados os objetivos, as metas, as preocupações e as ações potenciais para estabelecer os Elementos Primários de Avaliação (EPAs), que constituem-se de uma lista de ideias composta por objetivos, metas, ações, alternativas e valores dos decisores (BANA e COSTA, 1992). Os EPAs foram transformados em conceitos, sendo estes utilizados

5. Possua área mínima de $1.960 \mathrm{~m}^{2}$ e aloje entre $30.000 \mathrm{e}$ 150.000 aves. 
716 - Aplicação da Metodologia Multicritério de Apoio à Decisão no Relacionamento Interorganizacional na Cadeia da Avicultura de Corte

na construção de uma estrutura arborescente e cada conceito foi embasado teórica e empiricamente, a fim de eliminar as subjetividades e consolidar no modelo multicritério. Após, foi realizada uma reunião com os decisores (produtores rurais, gerente agropecuário e supervisor de fomento) para debater e selecionar os Pontos de Vista Fundamentais (PVF) e os Pontos de Vista Elementares (PVE) e compor uma estrutura arborescente. O estabelecimento da estrutura arborescente partiu do agrupamento dos conceitos, estabelecido via consenso entre os decisores. Os conceitos foram dispostos hierarquicamente, de acordo com as relações meios-fins, ou seja, na parte superior da estrutura constam os conceitos relacionados às metas, valores e objetivos dos decisores, sendo que estes deliberam sobre as características das ações desejadas, constituindo-se como Ponto de Vista Fundamental (PVF). E na parte inferior estão dispostas as ações necessárias para o cumprimento dos PVF, denominando-se como Pontos de Vista Elementares. Os pontos de vista traduzem os objetivos estratégicos em determinado cenário, pois especificam determinadas ações potenciais para tomada de decisão dos decisores entre uma gama de ações possíveis. Para finalizar esta primeira etapa da pesquisa realizou-se uma reunião com um pesquisador especialista na cadeia avícola, com o objetivo de discutir os resultados obtidos na reunião com os tomares de decisão e realizar ajustes do modelo.

Na segunda etapa, denominada de avaliação, foram utilizados os PVFs definidos na fase anterior para a construção de descritores. Estes, segundo Ensslin et al. (2001) "são conjuntos ordenados de níveis de impacto, organizados sobre uma escala [...] que servem para descrever o desempenho das ações potenciais associadas a um PVF" (p. 89). Constituem-se em uma função de valor que irá ordenar as preferências do decisor, avaliando ações potenciais, conforme determinado ponto de vista (ENSSLIN et al., 2001). Desta forma, os PVFs foram operacionalizados para a obtenção dos descritores, que constituíram os critérios, e assim, foi possível reconhecer a representatividade das ações a serem considera- das (REICHERT, 2012). Neste contexto, para evitar dualidade nos PVFs, a aplicação de princípios como o da mensurabilidade, operacionalidade e compreensibilidade se fizeram necessários para a validação dos descritores (KEENEY, 1992). Esta etapa foi realizada em agosto de 2014 e consistiu na aplicação dos dados e informações na construção do modelo para gerar os indicadores que foram utilizados para avaliar o relacionamento interorganizacional através da metodologia multicritério de apoio à decisão. Neste momento, os PVFs foram consolidados através da elaboração dos descritores e posterior transformação em critérios, para que fosse possível identificar o impacto das ações (ENSSLIN et al., 2001). Para estabelecer a preferência entre as alternativas foram utilizados os critérios, sendo necessário para sua construção um descritor e uma função de valor vinculada a cada descritor. Os descritores constituíram-se da ordenação de níveis de impacto, em escala decrescente de importância. Dentre os métodos discretos, o MAUT foi selecionado ${ }^{6}$, pois se distingue dos demais métodos multicritérios por tratar de problemas com objetivos conflitantes, denominados de atributos, além de apresentar diversos fatores qualitativos e quantitativos que influenciam a decisão. Os critérios são transformados em uma função de utilidade que, segundo Ensslin et al. (2001), são aplicadas para transformar os valores (quantitativos e qualitativos) associados aos critérios de cada alternativa em uma escala de dimensão comum. Por sua vez, a função de valor ordena de forma numérica as escolhas dos decisores, avaliando as ações potenciais, conforme seu ponto de vista (ENSSLIN et al., 2001). A partir das entrevistas com os decisores

\footnotetext{
6. Referente à classificação do método multicritério, esta divide-se em discretos, quando se trabalha com um número finito de alternativas, ou contínuos, quando o número de alternativas é infinito. Em função do número determinado de alternativas, foi utilizado o método discreto, que por sua vez engloba os métodos: MACBETH (Measuring Attractiveness by a Categorical Based Evaluation Technique), MAUT (Multi-Attribute Utility Theory), o AHP (Analytic Hierarchy Process), os métodos ELECTRA (Elimination et Choix Trafuisant la Realité) e os métodos PROMÉTHÉE (Preference Ranking Organization Method for Enrichment Evaluations).
} 
foram obtidos 14 descritores que foram apresentados individualmente aos decisores para que estes estabelecessem: (1) definição dos valores máximo (100) e mínimo (0); (2) valores descrentes nos níveis intermediários e (3) identificação da região de expectativa do descritor, auferindo níveis Bom e Neutro. Definidos os valores dos descritores foram constituídas as funções de valor locais referentes a cada nível de impacto, através do método Direct Rating descrito por Ensslin et al. (2001). Cumprida esta fase, um dos produtores integrados foi selecionado pelo grupo de produtores para harmonizar as respostas, sendo que sua seleção ocorreu por meio de votação pelos demais decisores. Posteriormente foi realizada uma reunião, no escritório do produtor, junto ao supervisor de fomento da empresa para buscar-se o consenso dos descritores. De posse dos descritores o facilitador efetuou a transformação linear da escala das funções de valor locais, onde fixou-se o valor 0 (zero) da escala no nível Neutro, e o valor 100 (cem) no nível Bom; desta forma, a região de expectativa foi determinada de 0 a 100 . Concluída esta etapa foi realizada a determinação de taxas de compensação ou taxa de substituição, em que são expressas as perdas de desempenho que uma ação potencial deve sofrer em um critério para compensar o ganho em outro de tal forma que o seu valor global permaneça inalterado, sendo estabelecido pelo método Swing Weights, descrito detalhadamente por Ensslin et al. (2001). Utilizou-se a função de agregação aditiva para compilar as diversas dimensões de avaliação das ações do modelo multicritério; assim, a ponderação de cada critério foi definida pelas taxas de substituição (ENSSLIN et al., 2001). Desta forma, conclui-se a elaboração do modelo multicritério.

Por fim, na terceira etapa, foi realizada a análise de competitividade da cadeia de suprimentos em função dos critérios apontados pelos decisores, obtendo-se as recomendações para os agentes econômicos. Primeiramente foi realizada a análise local, avaliando de forma independente cada um dos critérios do modelo multicritério. A seguir, os critérios individuais foram agregados, gerando um gráfico denominado perfil de impacto. De posse destas informações foi possível identificar as potencialidades das ações, bem como o desempenho em cada um dos critérios (REICHERT, 2012). A análise global, realizada na sequência, constituiu-se da parametrização de uma equação de agregação aditiva, que transforma as unidades de atratividade local em unidades de atratividade global. Assim, obteve-se a soma ponderada dos valores parciais relativo aos aspectos do relacionamento nos vários critérios, sendo realizada via taxa de substituição de cada critério (REICHERT, 2012). Para operacionalizar essa etapa foi realizada a determinação do perfil de impacto do modelo multicritério sobre três classes de produtores integrados, divididos conforme a remuneração obtida em 2013 na atividade avícola. Na classe A, $25 \%$ dos produtores obtiveram a melhor remuneração; na classe $\mathrm{B}$, $50 \%$ dos produtores obtiveram remuneração intermediária; e na classe C, 25\% dos produtores obtiveram a pior remuneração. No total, foram selecionados aleatoriamente no banco de dados da agroindústria integradora cinco produtores da classe A, 10 produtores da classe B e 5 da classe C. As entrevistas foram realizadas nas propriedades rurais com os proprietários das instalações, em setembro de 2014, a partir de questionário estruturado com perguntas sobre o custo de produção, remuneração, fatores de produção, risco de investimento, fluxo de informação e ambiente institucional. Para elucidação das respostas, as perguntas foram sistematizadas previamente e o entrevistado fez a escolha de alternativas dos diferentes critérios.

\section{Resultados e discussão}

Na Figura 1 apresenta-se a estrutura arborecente do modelo multicritério, reunindo os Pontos de Vista Fundamentais e os Pontos de Vista Elementares identificados na fase de estruturação. A estrutura da lista de conceitos (Elementos Primários de Avaliação - EPAs) levantada pelos decisores gerou seis grandes áreas de interesses, definidos como Pontos de Vista Fundamentais, 


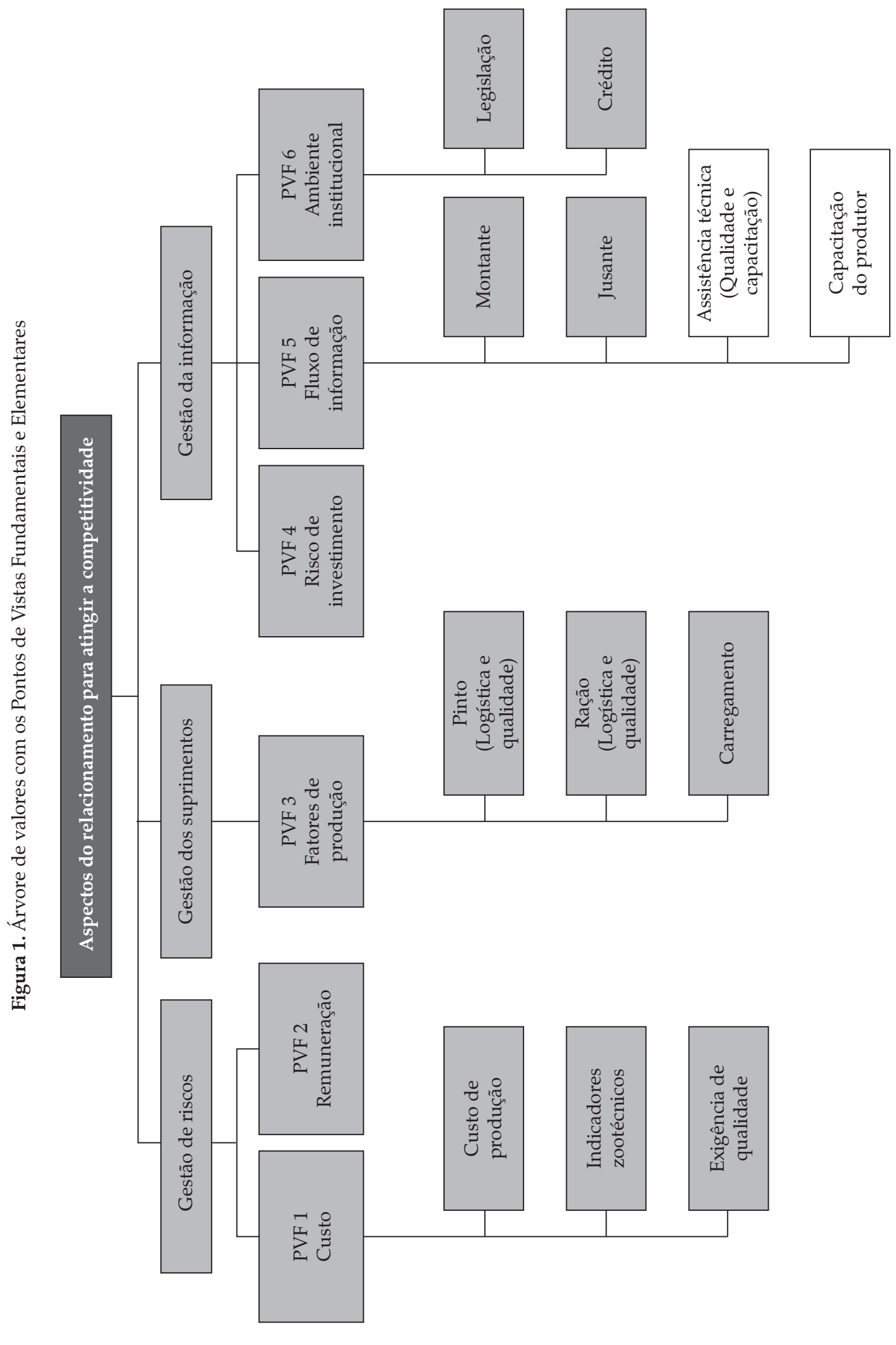


em ordem de preferência do decisor com relação ao impacto na competividade da cadeia de suprimentos (taxa de compensação normatizada): custo (22\%); remuneração (19\%); fatores de produção (17\%); risco de investimento (15\%); fluxo de informação (14\%) e ambiente institucional $(13 \%)$.

Dentre os critérios do modelo, o custo de produção foi o que apresentou maior peso para os agentes, isso porque é uma informação financeira indispensável para a avaliação do desempenho da atividade avícola, destacando-se o subcritério indicador zootécnico, por ser o item mais abordado pela assistência técnica. O subcritério "custo de produção" buscou avaliar o monitoramento das despesas na propriedade por meio da indagação se há o monitoramento sistemático dos custos na propriedade, ou seja, se durante a condução do lote são observadas as despesas e receitas, sendo tomadas ações proativas na correção de distorções. Também se ocorria, na época da pesquisa, a avaliação ao final de cada lote a partir do resultado consolidado informado pela integradora.

Outro critério em ordem de importância foi a remuneração paga ao produtor integrado, estando, assim, os dois itens de maior impacto relacionados à gestão de riscos. A remuneração dos produtores é uma forma de estimular os investidores a buscarem resultados superiores em suas granjas e se engajarem nos padrões de qualidade utilizados pela integradora. Aborda questões como a clareza dos contratos vigentes, da compatibilidade das exigências instituídas pela integradora em relação à remuneração paga e o efetivo cumprimento das regras pré-estabelecidas por ambas as partes. Dessa forma, a remuneração diz respeito ao desempenho dos produtores integrados, em que os critérios traçam um perfil da atividade em relação às metas estabelecidas pela empresa integradora, junto à clareza sobre as regras e o cumprimento dos requisitos de contrato para recebimento de bonificação pelo lote. Foi avaliada a clareza dos contratos quanto à remuneração, em um cenário de regras claramente especificadas, ou seja, não há dúvidas por parte dos integrados da forma de pagamento até uma condição em que há necessidade de melhorar a relação devido à obscuridade de alguma cláusula. A questão que indaga se a remuneração está condizente com o nível de exigência a ser atingida pela avicultura de corte busca mensurar a satisfação dos integrados quanto às requisições provenientes do mercado consumidor e sua remuneração, partindo de uma condição adequada em que há o equilíbrio entre exigências e os benefícios concedidos a uma condição em que precisa melhorar.

Os fatores de produção vinculados à gestão dos suprimentos foram o terceiro em ordem de importância por se tratarem de itens que possuem impacto direto sobre a produção como pintos, ração e carregamento, sendo o de maior importância para os decisores o subcritério ração, devido à sua importância no desenvolvimento das aves e representatividade nos custos de produção. O julgamento da qualidade do pinto entregue para alojamento e o processo logístico de entrega são fatores cruciais para o bom desempenho de um lote, em função dos impactos produtivos e fisiológicos sobre a uniformidade, ganho de peso e sanidade das aves. A possibilidade de avaliação e monitoramento do fornecimento de pintos, ração e carregamento gera segurança e confiança para a fase de engorda das aves. Assim, estas medidas que otimizam os processos e levam a uma assídua troca de informações remetem a um alinhamento na coordenação da cadeia, proporcionando o desenvolvimento e a efetivação de práticas que transmitam um aumento da qualidade, da logística e do sistema de informação (VAN DER VORST et al., 2005).

A análise do risco de investimento retrata as subjetividades relacionadas ao setor e ao negócio, seja a montante ou a jusante na cadeia de suprimentos. Desta feita avaliou-se a confiança para investir na atividade, as garantias contratuais relacionadas ao investimento e o compartilhamento dos riscos entre integrado e integradora. O PVF "risco de investimento" retrata a insegurança do produtor integrado referente às exigências e ao atendimento aos mercados e seus 
desdobramentos sobre a atividade. Considera as barreiras comerciais impostas pelos países importadores, o risco sanitário da possível entrada no País de doenças de notificação obrigatória, como a gripe aviária e new casttle, além da perda de mercado pela empresa integradora. Foi avaliada a confiança do integrado para investir na atividade, mesmo sobre um cenário de insegurança. O produtor foi questionado se está seguro para investir ou se precisa de garantias por parte da integradora para entrar ou expandir a atividade. Da mesma forma, buscou-se a informação se o contrato de parceria de alguma forma garante a vida útil do investimento, ou seja, se contraído um investimento para a atividade, durante seu vigor se é garantido o alojamento de aves.

O Ponto de Vista Fundamental "Fluxo de informação" foi considerado como quinto em ordem de importância para os decisores. Este critério aborda a organização dos produtores rurais para interlocução com a integradora e os prestadores de serviço. Está subdividido nos subcritérios assistência técnica, jusante e montante, sendo o subcritério assistência técnica o que apresentou maior representatividade em função do contato periódico e estreito relacionamento entre produtores e extensionistas. A existência de assimetrias de informação na cadeia de suprimentos, as quais podem ser geradas pelo comportamento oportunista dos agentes envolvidos na transação demanda a implementação de um mecanismo eficiente de coordenação. Destaca-se como resultado que os critérios que dizem respeito à gestão da informação possuem menor importância que os critérios vinculados à produção. A gestão da informação possui estreita relação com a competitividade pela possibilidade de mensuração e monitoramento, como ocorre para os principais insumos da avicultura: pinto, ração, medicamentos etc., que geram indicadores como conversão alimentar, peso e mortalidade, garantindo, assim, informações diferenciadas, que poderão ser utilizadas para obter vantagens sobre os concorrentes. Ademais, as propriedades rurais estão alinhadas à visão corporativa por meio da adoção de mecanismos de coordenação, como a padroni- zação nas instalações que aumentam a escala de produção e as adoções tecnológicas para aumentar a eficiência dos processos que se constituem numa importante componente para a alavancagem de custos.

O fluxo de informação que ocorre a "montante" da cadeia de suprimentos busca compreender as inter-relações entre produtores integrados e prestadores de serviços, como a agroindústria integradora, empresa de carregamento, fornecedores de equipamentos, maravalha, lenha e manutenção das instalações, apontando possíveis conflitos. À exceção da integradora e da empresa de carregamento, nos demais casos não há formalização contratual com os fornecedores quanto a prazo, qualidade ou garantia de entrega, sendo estabelecidas com base na confiança entre as partes, em função da frequência de negociação.

Por fim, o critério "Ambiente institucional", que apresentou a menor taxa de compensação normativa $(0,13)$, está relacionado à situação da atividade quanto à influência da legislação, seja ambiental, sanitária e trabalhista e a política de crédito entre o produtor integrado e a integradora. $\mathrm{O}$ ambiente institucional diz respeito às principais instituições que influenciam as regras que condicionam as atividades da cadeia produtiva, as relações econômicas, sociais e ambientais e os padrões de qualidade dos produtos.

\subsection{Determinação do perfil de impacto por classe de desempenho}

A determinação do perfil de impacto de acordo com a classe de remuneração foi utilizada como forma de se parametrizar o modelo multicritério. A primeira classe representa $25 \%$ dos produtores integrados que obtiveram a melhor remuneração em 2013, sendo denominados de "classe A". Os produtores com renda intermediária e que representam $50 \%$ da população foram denominados de "classe B". Por fim, $25 \%$ dos produtores com pior renda em 2013 foram chamados de "classe C". Para cada critério e subcritério foram construídos descritores. Nestes, os decisores identificaram o nível de impacto que melhor 
descrevia sua atividade. A partir da determinação do nível de impacto, definiu-se, por meio das escalas de valores cardinais, quantos pontos a classe obteve no ponto de vista (Figura 2).

A comparação das pontuações obtidas pelas três classes avaliadas com os níveis Bom e Neutro permite posicionar cada classe frente às expectativas dos produtores integrados e da agroindústria integradora, entre uma condição de atratividade (Bom) e uma condição em que não há atratividade nem repulsividade (Neutro). Contudo, não foi considerada uma avaliação comparativa entre as três classes, em função de o objetivo ser a proposição de estratégias a partir do seu perfil de impacto para melhorar suas capacidades de competição.

Os produtores da classe A apontaram que a evolução de uma condição atual para a realização de ações potenciais teria maior efeito sobre os critérios: remuneração (42,75 pontos), risco de investimento (35 pontos), jusante ( 16,33 pontos), custo de produção (7,25 pontos), capacitação do produtor (5,74 pontos), ração (5,38 pontos), carregamento (2,55 pontos), exigência de qualidade ( 1,65 ponto) e pinto ( 1,55 ponto). Para os produtores da classe $\mathrm{B}$, a ordem dos critérios foi: remuneração ( 47,5 pontos), risco de investimento
(35 pontos), jusante (16,33 pontos), custo de produção (11,6 pontos), capacitação do produtor (5,74 pontos), indicadores zootécnicos (5,43 pontos), ração (5,38 pontos), exigências de qualidade (4,12 pontos), montante (3,36 pontos), legislação (2,72 pontos), carregamento (2,55 pontos) e pinto (1,55 ponto). Por fim, os produtores da classe C consideraram os critérios na seguinte ordem: risco de investimento (35 pontos), remuneração (19 pontos), jusante (16,33 pontos), custo de produção (14,5 pontos), carregamento ( 7,65 pontos), capacitação do produtor (5,74 pontos), pinto $(5,44$ pontos), legislação (3,39 pontos) e exigências de qualidade (1,65 ponto).

Para o subcritério "custo de produção" apenas a classe A dos produtores ficou no nível Neutro, sendo que as classes B e C ficaram bem distantes da região de expectativa, mostrando que as informações coletadas não são utilizadas para a discussão entre integrado e integradora. Já no subcritério "indicadores zootécnicos" apenas a classe A ficou acima do nível Bom; os demais ficaram dentro da região de expectativa, indicando que há o monitoramento sistemático dos indicadores pelos agentes da cadeia. Quanto à "exigência de qualidade", as três classes ficaram dentro da região de expectativa, próximas ao nível Bom,

Figura 2. Nível de impacto por classe de produtor

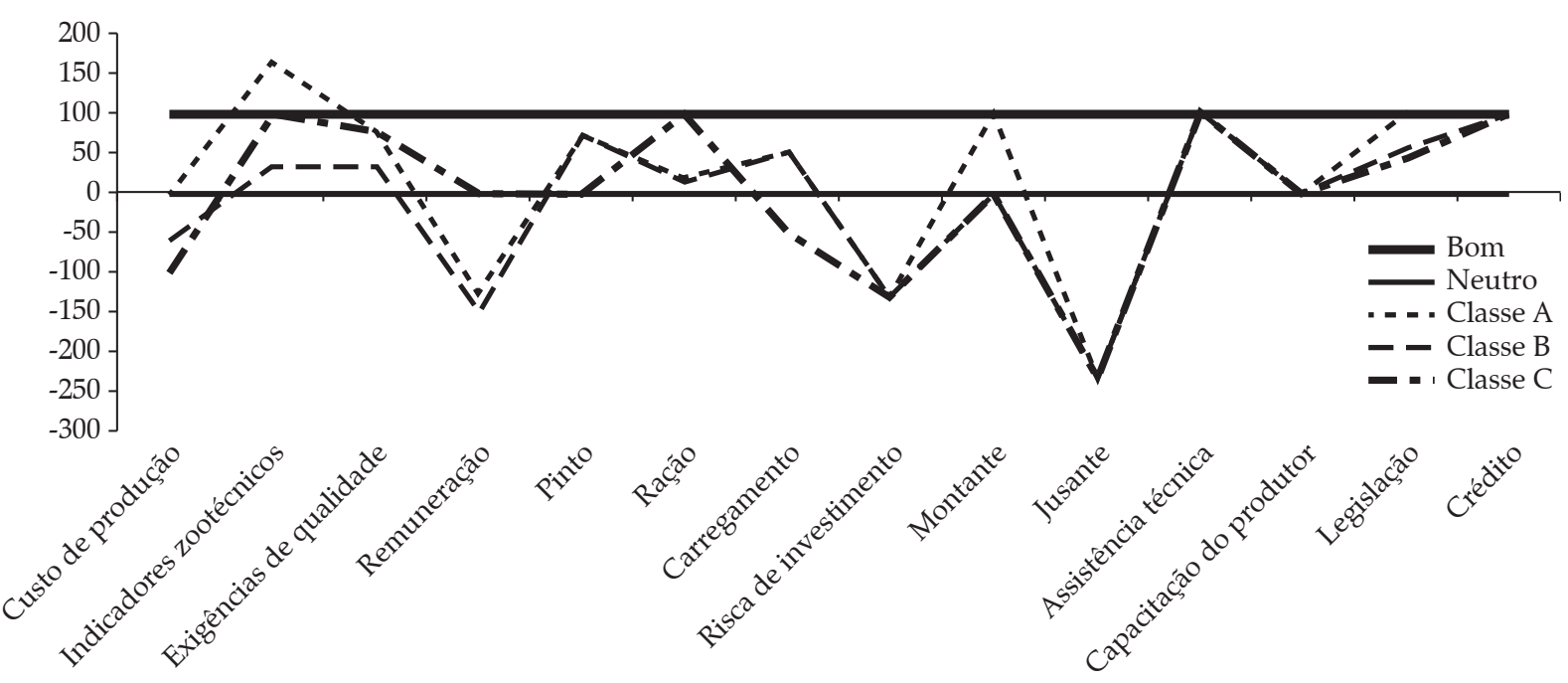

Fonte: Dados da pesquisa (2014). 
mostrando que há o monitoramento sistemático dos padrões de qualidade. Assim, apenas o subcritério "custo de produção" necessita de ações efetivas para melhorar o resultado, tendo em vista que este pode ser um indicador para auxiliar na explicação da remuneração e dos riscos de investimentos da atividade.

No critério "remuneração", apenas a classe C ficou no nível Neutro, indicando que precisa melhorar a remuneração e o cumprimento dos requisitos do contrato. Já para as classes A e B acrescenta-se a questão que o contrato de parceria não possui regras claras para a remuneração. Chama a atenção o fato de as classes A e B serem mais exigentes que a classe $\mathrm{C}$ quanto aos fatores ligados à remuneração, implicando em ações que tornem o contrato mais claro. Ressalta-se que é importante e necessário discutir com os produtores sobre o custo de produção para se chegar a um denominador para a remuneração. Nos subcritérios "pinto" e "ração", todas as classes ficaram dentro da região de expectativa. Já no subcritério "carregamento" apenas a classe $\mathrm{C}$ ficou fora, mostrando que a previsão de carregamento e o custo da atividade precisam melhorar. Este subcritério possui importância em função de o contrato ser com uma empresa terceirizada e ocorrerem reajustes nos pagamentos periodicamente, sendo um motivo de conflito.

Quanto ao "risco de investimento", as três classes ficaram fora da região de expectativa, apontando que há necessidade da apresentação de garantias para investir na atividade e o contrato não garante a vida útil do investimento. Este critério é específico da integração estudada, em decorrência da frequente troca no comando da agroindústria, o que gerou insegurança na contração de novos investimentos.

O fluxo de informação a "montante" da cadeia para as classes A e C atingiu o nível Bom, ao passo que a classe $B$ permaneceu no nível Neutro. Por sua vez, o fluxo de informação a "jusante" da cadeia, para as três classes, encontra-se fora da região de expectativa, demonstrando baixo empenho no suprimento de informações referentes à comercialização dos produtos ori- ginados da matéria-prima. O subcritério "assistência técnica" ficou dentro do nível Bom para as três classes. E a "capacitação dos produtores" apontou nas três classes que os treinamentos gerenciais precisam melhorar. $\mathrm{O}$ critério fluxo de informação transparece a necessidade de maior articulação e coordenação na comunicação entre os agentes, seja nas informações a jusante da cadeia, como na capacitação fornecida pela agroindústria, mostrando uma demanda por parte dos produtores por informações dos processos que fazem parte.

O subcritério "legislação" apresentou-se nas três classes dentro da região de expectativa, em que o rigor da legislação não altera a intenção de investir na atividade. No subcritério "crédito", as três classes posicionaram-se no nível Bom, em que as linhas de créditos são adequadas para investir na atividade, há o aval da integradora para a captação de recursos e não se considera importante o compartilhamento do gerenciamento dos financiamentos. Neste sentido, o critério ambiente institucional mostrou-se em conformidade com o arranjo (produtores e agroindústria).

\subsection{Ações potenciais a partir do modelo multicritério}

Abaixo do nível Neutro estão 35\% dos critérios e subcritérios, em que se destacam para os produtores classe A: "remuneração", "risco de investimento" e informações a "jusante" da cadeia. Para a classe B destacam-se: "custo de produção", "remuneração", "risco de investimento" e informações a "jusante" da cadeia. Já para a classe C obteve-se como resultado: "custo de produção", "remuneração", "carregamento", "risco de investimento" e informações a "jusante" da cadeia.

$\mathrm{O}$ "custo de produção" teve baixo desempenho nas três classes de produtores, ficando fora da região de expectativa nas classes B e C; ou seja, a execução de ações geraria maior impacto. Para $40 \%$ e $80 \%$ dos produtores da classe A e B, respectivamente, o monitoramento das informações de custo de produção são realizadas ao final de cada lote, ao passo que $60 \%$ dos produtores da classe C 
não efetuam nenhum tipo de monitoramento das receitas e despesas.

No tocante ao diálogo sobre o desempenho na propriedade, $40 \%$ dos produtores da classe A, 70\% da classe B e $60 \%$ da classe C não trocam informação com a agroindústria sobre custos de produção. Segundo alguns relatos, o principal motivo relaciona-se à falta de abertura para o diálogo. Assim, a adoção de ações potenciais sobre o custo de produção causaria um impacto global de 7,25 pontos para a classe A, 11,6 pontos para a classe B e 14,5 pontos para a classe C. Neste cenário, poder-se-ia apontar como recomendações: 1) a implantação da ferramenta gerencial de fluxo de caixa em todas as granjas; 2) o treinamento e habilitação da equipe técnica para lidar com as informações disponíveis a campo; 3) incentivar a associação dos produtores a coletar informações referentes ao resultado dos lotes, como forma de obter um índice de referência e 4) reuniões anuais de discussão entre a agroindústria e os produtores.

O critério "remuneração" apresentou-se fora da região de expectativa para todas as classes de produtores, sendo o critério que apresenta maior resposta quando aplicadas ações potenciais. Para $80 \%$ das classes A e B e $40 \%$ da classe C, o contrato de integração não possui regras claras para a remuneração, principalmente no que diz respeito à aplicação dos indicadores zootécnicos como conversão alimentar, mortalidade e peso nas fórmulas que estabelecem o valor. Quando questionados sobre a paridade no cumprimento das exigências do contrato entre os produtores integrados e agroindústria integradora, $40 \%$ do público classe A, $50 \%$ da classe B e $60 \%$ da classe $\mathrm{C}$ consideram que a relação precisa melhorar, $\mathrm{O}$ que seria um indicativo de que, para produtores, as obrigações do cumprimento do contrato tendem a ser desproporcionais entre os agentes da cadeia.

Por fim, sobre a remuneração paga por lote de frango, para $80 \%$ dos produtores da classe A e B e $100 \%$ da classe $C$ a remuneração não está compatível com as exigências a serem atendidas, como adequação tecnológica, biosseguridade, gestão, entre outros fatores. Um argumento citado é que não há correção da tabela de remuneração pelos índices de inflação. Esta questão merece atenção, em função de que, independente da remuneração paga há um sentimento de insatisfação, o que sugere que mais importante que o valor é a clareza para os agentes sobre a rentabilidade e os custos da atividade como um todo.

O benefício esperado com a execução de ações potenciais sobre o critério remuneração será de 42,75 pontos para a classe A, 47,5 pontos para a classe $\mathrm{B}$ e 19 pontos para a classe $\mathrm{C}$. O critério remuneração foi o que apresentou maior impacto global, ou seja, a execução de medidas proporcionaria resultados mais significativos sobre o relacionamento, sendo possíveis as seguintes recomendações: 1) reuniões gerenciais entre produtores e integradora para tornar mais transparente as regras do contrato de parceria; 2) investigar as possíveis causas de desalinhamento de responsabilidades no cumprimento do contrato; 3) abordar em discussões entre integrados e integradora sobre a rentabilidade da avicultura de corte.

O subcritério "carregamento" apresentou resultados distintos entre as classes, e a $\mathrm{C}$ apresentou desempenho negativo; desta forma, a realização de ações terá maior impacto. As classes A e B possuem desempenho intermediário em relação a um desempenho potencial. Os produtores da classe $C$ apresentaram baixa pontuação, indicando que para $60 \%$ dos entrevistados, no ano, os lotes não foram carregados na data prevista. Esta informação é divergente das classes A e B, que obtiveram $80 \%$ e $90 \%$, respectivamente, carregados na data prevista. Assim, esta insatisfação com o carregamento pode ser decorrente do mau desempenho acumulado dos lotes de frango da classe de produtores. O procedimento de carregamento foi considerado adequado para $60 \%$ do público-alvo. Contudo, o valor pago à empresa terceirizada que realiza o carregamento precisa melhorar para $80 \%$ dos entrevistados. Este posicionamento é similar ao encontrado nas classes A e B, que obtiveram $100 \%$ e $90 \%$ de insatisfação com o valor, respectivamente. $\mathrm{O}$ índice de 
avaliação para o subcritério carregamento foi de 2,55 pontos para as classes A e B e 7,65 pontos para a classe C. As recomendações para o subcritério carregamento são: 1) transferência do contrato de carregamento dos integrados para a agroindústria integradora, em função de seu poder de barganha; 2) criar cláusulas de desempenho ligadas à remuneração para as equipes de carregamento; 3) reuniões anuais para a discussão entre produtores integrados e prestadora de serviço sobre as responsabilidades e benefícios do contrato.

No critério "risco de investimento", as três classes ficaram fora da região de expectativa, tendo o segundo critério maior peso quando realizadas ações potenciais. Quando questionados sobre a segurança para investir na atividade, $80 \%$ dos produtores da classe A, 90\% da classe B e $100 \%$ da classe C afirmaram que necessitam de mais garantias da agroindústria integradora para investir na atividade. Da mesma forma, foi consenso a necessidade de que o contrato de integração deva garantir a vida útil do investimento, sendo que $60 \%$ da classe A, 70\% da classe B e $100 \%$ da classe C concordam com esta afirmativa. Observa-se que quanto menor for a remuneração, maior tende a ser a necessidade de respaldo da empresa integradora. Por fim, para $60 \%$ das classes A e C e $80 \%$ da classe B há o devido compartilhamento dos riscos de investimento entre produtores integrados e agroindústria integradora.

Neste sentido, a adoção das ações potenciais sobre riscos de investimento resultaria em 35 pontos para as três classes estudadas. Este critério foi o segundo item com maior impacto no modelo multicritério, sendo que para correção desta lacuna propõe-se: 1) disponibilização de informações sobre as transações comerciais e mercados da empresa integradora; 2) investigar as variáveis que levam à insegurança para investir na atividade; 3) dialogar sobre os principais riscos de investimento compartilhados; 4) elaboração pela assistência técnica e produtores integrados de um plano de ação estratégico anual para cada granja, com metas para os principais indicadores zootécnicos (conversão alimentar, perdas por condenações, calo de pata etc.) e alinhamento de objetivos em comum.

O fluxo de informações a "jusante" da cadeia de suprimento foi um ponto deficitário na opinião das três classes de produtores. Para $80 \%$ da classe A, $90 \%$ da classe B e $100 \%$ da classe C não ocorre o compartilhamento de informação na frequência esperada entre os agentes. Da mesma forma, para $60 \%$ das classes A e C e 70\% da classe $\mathrm{B}$, quando se tem contato com notícias ou informações a respeito das transações da agroindústria integradora, estas não transmitem segurança e qualidade. Estes baixos níveis de confiança são explicados por fatores contextuais, como: a assimetria de poder, má comunicação, baixos níveis de cooperação e a falta de informação (LINDGREEN et al., 2005).

A execução de medidas sobre a troca de informações a jusante da cadeia de suprimentos geraria um impacto global de 16,33 pontos para as três classes. O subcritério jusante foi o que apresentou pior desempenho atual para as três classes de produtores, tendo como sugestões para melhor articulação: 1) institucionalizar um programa de fluxo de informação sobre a comercialização e seus impactos para a cadeia produtiva no âmbito nacional, regional e local; 2) discutir sobre a conjuntura nacional e internacional do setor com os produtores integrados; 3) envolver a associação de produtores e utilizar ambientes como fóruns regionais e locais para veicularem informações sobre a cadeia de suprimentos.

\section{Conclusão}

Conclui-se que os agentes da cadeia de suprimentos da avicultura de corte estudada, dependendo da classe em que os produtores se enquadram, se utilizam de critérios diferenciados para tomada de decisão que influenciam o relacionamento e a competitividade do sistema.

Os produtores da classe A apresentaram três diferentes níveis de impactos às respostas, sendo remuneração e risco de investimento enquadrados como de alta prioridade. Isso sugere que 
nestes critérios há assimetria de informação entre os agentes, constituindo um ambiente de incerteza no relacionamento. Os critérios e subcritérios como custo de produção, capacitação do produtor, ração, carregamento, exigência de qualidade e pinto apresentaram menor importância para os decisores. Por fim, indicadores zootécnicos, montante, assistência técnica, legislação e crédito não se destacaram no modelo.

Os resultados para os produtores da classe $B$ foram similares ao da classe $A$, em que se observaram três diferentes níveis de impacto nas respostas, mantendo os critérios remuneração e risco de investimento enquadrados como de alta prioridade. Em nível intermediário de importância destacaram-se os critérios fluxo de informação a jusante da cadeia de suprimentos e custo de produção. Apresentado menor prioridade pelo decisores enquadrados na classe $B$, foram identificados a capacitação do produtor, indicadores zootécnicos, ração, exigência de qualidade, montante, legislação, carregamento e pinto. Já os subcritérios assistência técnica e crédito não apresentaram impacto no modelo.

Por fim, os resultados dos produtores da classe $C$ tiveram em um primeiro nível de prioridade apenas o critério risco de investimento e, em nível intermediário, a remuneração, jusante e custo de produção, indicando que a classe $C$ concentra as tomadas de decisão nos critérios de gestão de riscos. Em nível mais baixo de prioridade para esta classe foram identificados o carregamento, capacitação do produtor, pinto, legislação e exigência de qualidade. Os critérios e subcritérios indicadores zootécnicos, ração, montante, assistência técnica e crédito não apresentaram retorno à execução de ações potenciais.

Quando comparados os dados entre as classes de produtores, as ações sobre o critério remuneração apresentaram mais que o dobro de eficácia para as classes A e B em relação à classe $C$, o que mostra que há maior desconfiança destas classes no processo e na relação contratual relacionado à remuneração. $\mathrm{O}$ critério risco de investimento apresentou o mesmo resultado para as três clas- ses de produtores e aponta grande diferença nas respostas dos entrevistados. Isso se deve principalmente à insegurança para investir na atividade e à ausência de garantias no contrato referente ao investimento. O fluxo de informação a jusante da cadeia de suprimentos apresentou resultados idênticos para as três classes de produtores, visto que não há o compartilhamento de informação na frequência esperada, além das informações não transmitirem segurança e qualidade. Para o subcritério custo de produção, as ações potenciais são mais efetivas para as classes C, B e A, respectivamente, possuindo $100 \%$ de diferença entre as classes extremas, o que demonstra que, quanto pior a renda, menor é o diálogo entre produtores integrados e integradora. Possivelmente, esse resultado reflete o desgaste que há na relação à medida que a remuneração é afetada. No subcritério carregamento, as ações potenciais apresentam maior resultado para a classe $\mathrm{C}$ em relação às classes A e B. Isso ocorre principalmente devido à insatisfação com a data de carregamento, que pode ter como justificativa o mau desempenho acumulado dos lotes, uma vez que a avaliação do procedimento foi considerada adequada pelas demais classes. O subcritério ração apresenta problemas esporádicos de qualidade e entrega e os benefícios da realização de ações potenciais são apenas para as classes A e B o que mostra que este público é mais exigente. Por sua vez, no subcritério pinto, a situação se inverte e o nível de exigência concentra-se sob a classe $C$, sendo duas vezes maior que as demais classes.

Em decorrência das limitações de tempo e recursos financeiros, a pesquisa foi conduzida em apenas uma agroindústria integradora, na microrregião de Seara, Santa Catarina. Assim, esta ferramenta metodológica expressa a realidade somente dos envolvidos com esta empresa. Tendo em vista que esta metodologia complementa os conceitos teóricos supracitados, sugere-se que esta seja utilizada para realização de estudos em outras integradoras e regiões, permitindo melhor definição do relacionamento entre os agentes na cadeia de suprimentos da avicultura de corte. 


\section{Referências}

ALMEIDA, D. F. Análise multicritério no mercado de futuro: seleção de fruta. 2011. 108 f. Dissertação (Mestrado) - Programa de Pós-graduação em Engenharia da Produção, Universidade Federal de Pernambuco, Recife, 2011.

ANSOFF, I. Estratégia Empresarial. São Paulo: McGrawHill, 1977. 203 p.

ARBAGE, A. P. Custos de transação e seu impacto na formação e gestão da cadeia de suprimentos: estudo de caso em estruturas de governança híbridas do sistema agroalimentar no Rio Grande do Sul. 2004. 280 f. Tese (Doutorado) - Programa de Pós-Graduação em Administração, Escola de Administração, Universidade Federal do Rio Grande do Sul, Porto Alegre, 2004.

AREND, M. R. et al. Múltiplos Critérios de Apoio à Decisão em SIG para a Promoção de Sistemas Orgânicos de Produção Agropecuária no Município de Arroio do Meio - RS. In: SIMPÓSIO BRASILEIRO DE SENSORIAMENTO REMOTO, 15., 2011, Curitiba. Resumo. Curitiba: SBSR, 2011.

ARONDEL, C. e GIRARDIN, P. Sorting cropping systems on the basis of their impact on groundwater quality. European Journal of Operational Research, Amsterdam, v. 127, p. 467-482, 2000.

BALVERDE, N. R. M. Desenvolvimento de uma ferramenta de apoio ao processo de negociação integrativa. 2006. $159 \mathrm{f}$. Tese (Doutorado) - Programa de Pós-graduação em Engenharia da Produção, Universidade Federal de Santa Catarina, Florianópolis, 2006.

BANA e COSTA, C. A. Structuration, Construction et Exploitation d'un Modele MCDA. 1992. 378 f. Tese (Doutorado) - Instituto Superior Técnico, Universidade Técnica de Lisboa, Lisboa, 1992.

BARNEY, J. B. Firm, resources and sustained competitive advantage. Journal of Management, Texas, v. 17, n. 1, p. 99-120, 1991.

BATALHA, M. O. e SOUZA FILHO, H. M. Analisando a Competitividade de Cadeias Agroindustriais: uma proposição metodológica. In: BATALHA, M. O. e SOUZA FILHO, H. M. (Org.). Agronegócio no MERCOSUL: uma agenda para o desenvolvimento. São Paulo: Atlas, 2009. p. 1-22.

BELARMINO, L. C. et al. Identificação de oportunidades de inovação em APL por multicritério. In: CONGRESSO BRASILEIRO DE ECONOMIA E SOCIOLOGIA RURAL, 49, 2011, Belo Horizonte. Resumo. Belo Horizonte: SOBER, 2011.
BIJMAN, J. Essays on agricultural co-operatives: Governance structure in fruit and vegetable chains. 2002. 194 f. Tese (PhD) - Erasmus University Rotterdam, Rotterdam, 2002.

COASE, R. H. The firm, the market and the law. Chicago: The University of Chicago Press, 1988.

DOROW, R. Coordenação e governança: um estudo de caso na cadeia de malacocultura da grande Florianópolis. 2013. 236 f. Dissertação (Mestrado) - Programa de PósGraduação em Agronegócios, Centro de Estudos e Pesquisas em Agronegócios, Universidade Federal do Rio Grande do Sul, Porto Alegre, 2013.

DUTRA, A. et al. Inovação no processo de avaliação do desempenho organizacional: o uso da dimensão integrativa. Revista de Administração e Inovação, São Paulo, v. 5, n. 2, p. 150-163, 2008.

ENSSLIN, L., MONTIBELLER NETO, G. eNORONHA, S. Apoio à decisão - metodologia para estruturação de problemas e avaliação multicritério de alternativas. Florianópolis: Insular, 2001. 296 p.

FARINA, E. M. M. Q., AZEVEDO, P. F. e SAES, M. S. M. A experiência de regulamentação de sistemas agroindustriais no Brasil, In: FARINA, E. M. M. Q., AZEVEDO, P. F. e SAES, M. S. M. (Org.). Competitividade: mercado, estado e organizações. Piracicaba: Ed. Singular, Fapesp/Pensa, 1997, p. 207-214.

FERRAZ, J. C., KUPFER, D. e HAGUENAUER, L. Made in Brasil: desafios competitivos para a indústria. Rio de Janeiro: Campus, 1995. 386 p.

FURLANETTO, E. L. Formação das estruturas de coordenação nas cadeias de suprimentos: estudos de caso em cinco empresas gaúchas. 2002. 306 f. Tese (Doutorado) - Programa de Pós-graduação em Administração, Escola de Administração, Universidade Federal do Rio Grande do Sul, Porto Alegre, 2002.

GENERINO, R. C. M. Contribuição da abordagem multicritério na seleção de alternativa de reuso de água: aplicação de um caso de irrigação agrícola e paisagística no Distrito Federal. 2006. 202 f. Tese (Doutorado) - Programa de Pós-graduação em Saúde Pública, Universidade de São Paulo, São Paulo, 2006.

GOMES, M. C. Apoio à decisão em empresas familiares em processo de evolução: um modelo multicritérios em um estudo de caso na indústria de conservas de Pelotas-RS. 2001. 417 f. Tese (Doutorado) - Programa de Pósgraduação em Engenharia da Produção, Universidade Federal de Santa Catarina, Florianópolis, 2001.

GOMES, E. G., MELLO,J.C.C.B.S. de eMANGABEIRA, J. A. de C. Índice multicritério de bem estar social 
rural em um município da região amazônica. Pesquisa Operacional, Rio de Janeiro, v. 28, n. 1, p. 141-160, 2008.

GUANZIROLI, C. E., SOUZA FILHO, H. M. e BUAINAIN, A. M. Metodologia para estudo das relações de mercado em sistemas agroindustriais. Brasília: IICA, 2008.

KALOGERAS, N. et al. Evaluating the financial performance of agri-food firms: a multicriteria decision-aid approach. Journal of Food and Engineering, Breukelen, v. 70, n. 1, p. 365-371, 2005.

KATZ, R. L. Cases and Concepts in Corporate Strategy. New Jersey: Prentice-Hall Inc., 1970. 341 p.

KEENEY, R. L. Value-focused thinking: a path to creative decision making. Cambridge MA: Harvard University Press, 1992. $416 \mathrm{p}$.

LAMBERT, D. R. e COOPER, M. C. Issues in supply chain management. Industrial Marketing Management, New York, v. 29, n. 1, p. 65-83, 2000.

LAZZARINI, S. G., CHADDAD, F. R. e COOK, M. L. Integrating supply chain and network analyses: The study of netchains. Journal Chain and Network Science, Wageningen, v. 1, n. 1, p. 1-21, 2001.

LINDGREEN, A., PALMER, R. e TREINEKENS, J. Relationship within the supply chain: A case study. Journal Chain and Network Science, Wageningen, v. 5, p. 85-99, 2005.

LOYCE, C., RELLIER, J. P. e MEYNARD, J. M. Management planning for winter wheat with multiple objectives: the Betha System. Agricultural Systems, Barking, v. 72, p. 9-31, 2002.

MARTINS, F. M. et al. Modelo multicritério para avaliação do potencial de negócios tecnológicos na agricultura. Cadernos de Ciência e Tecnologia, Brasília, v. 28, n. 1, p. 189-222, 2011.

MASTRANTONIO, J. J. da S., PORTO, R. G. e GOMES, M. C. A escolha de cultivares de feijão através de um modelo multicritério baseado no saber local. Revista Brasileira de Agroecologia, Cruz Alta, v. 2, n. 1, p. 694-697, 2007.

MAZZETTO, F. e BONERA, R. Meacros: a tool for multi-criteria evaluation of alternative cropping systems. European Journal of Agronomy, Amsterdam, v. 18 , p. 379-387, 2003.

NEVES, E. M. e NEVES, M. F. O agronegócio, desenvolvimento brasileiro e a importância da pesquisa. In: NEVES, M. F.(Org.) Agronegócios \& Desenvolvimento Sustentável: uma agenda para a liderança mundial na produção de alimentos e bioenergia. São Paulo: Atlas, 2011. p. 24-39.
PORTER, M. Competitive Advantage: creating and sustaining superior performance. New York: The Free Press, 1985. 321 p.

REICHERT, L. J. Avaliação de sistemas de produção de batata orgânica em propriedades familiares: uma aplicação da metodologia multicritério de apoio à decisão (MCDA). 2012. 346 f. Tese (Doutorado) - Programa de PósGraduação em Sistemas de produção Agrícola Familiar, Faculdade de Agronomia Eliseu Maciel, Universidade Federal de Pelotas, Pelotas, 2012.

ROY, B. Decision science or decision-aid science? European Journal of Operational Research, North-Holland, v. 66, p. 184-203, 1993.

ROY, B. Multicriteria methodology for decision aiding. Boston: Kluwer Academic Publishers, 1996. 292 p.

. e VENDERPOOTEN, D. The European school of MCDA: emergences, basic features and current works. Journal of Multi-Criteria Decision Analysis, London, v. 3, p. 23-38, 1996.

SAES, M. S. M. Diferenciação e apropriação da quase-renda na agricultura: a cafeicultura de pequena escala. 2008. $162 \mathrm{f}$. Tese (Livre Docência) - Departamento de Administração, Faculdade de Economia, Administração e Contabilidade, Universidade de São Paulo, São Paulo, 2008.

SILVA, C. A. e BATALHA, M. O. Competitividade em sistemas agroindustriais: metodologia e estudo de caso. In: WORKSHOP BRASILEIRO DE GESTÃO DE SISTEMAS AGROALIMENTARES, 2, 1999, Ribeirão Preto. Resumo. Ribeirão Preto: PENSA/FEA/USP, 1999.

STEVENS, G. C. Integrating supply chain. International Journal of Physical Distribution \& Materials Management, London, v. 19, n. 8, p. 3-8, 1989.

TRIENEKENS, J. H. et al. Transparency in complex dynamic food supply chains. Advanced Engineering Informatics, Wageningen, v. 26, p. 55-65, 2012.

Van der VORST, J. A. G. J. Performance measurement in agri-food supply chain networks. An overview. In: QUANTIFYING the Agri-Food Supply Chain. Dordrecht: Springer, 2005.

e BEULENS, A. J. M. Identifying sources of uncertainty to generate supply chain redesign strategies. International Journal of Physical Distribution \& Logistics Management, Wageningen, v. 32, n. 6, p. 409-430, 2002.

VAN DUREN, E., MARTIN, L. e WESTGREN, R. Assessing the Competitiveness of Canada's Agrifood Industry. Canadian Journal of Agricultural Economics, Toronto, v. 39, p.727-738, 1991.

WILLIAMSON, O. E. The new institutional economics: Taking stock, looking ahead. Journal of Economic Literature, New York, v. 38, p. 595-613, 2000. 
728 - Aplicação da Metodologia Multicritério de Apoio à Decisão no Relacionamento Interorganizacional na Cadeia da Avicultura de Corte

Transaction cost economic and organizational

theory. Journal of Industrial Corporate Change, New York, v. 2, p. 107-156, 1993.

XAVIER, J. H. V. et al. Metodologia multicritério de apoio à decisão como ferramenta para avaliação de sistemas de cultivo de milho. Cadernos de Ciência e Tecnologia, Brasília, v. 29, n. 1, p. 89-131, 2012.
YIN, R. K. Estudo de Caso: planejamento e métodos. 2 ed. Porto Alegre: Brookman. 2001.

ZYLBERSZTAJN, D. Estruturas de governança $e$ coordenação do agribusiness: uma aplicação da nova economia das instituições. 1995. 241 f. Tese (Livre docência)-Departamento de Administração, Faculdade de Economia, Administração e Contabilidade, Universidade de São Paulo, São Paulo, 1995. 